\section{SHORT-TERM ORTHODONTICS: HIGH PROFITABILITY AND LOW RISK}

Sir, much has been written, ${ }^{1}$ contested ${ }^{2}$ and debated ${ }^{3}$ over the issue of short-term cosmetic orthodontics for the GDP. At the BDA Conference in Manchester in April, there were three presentations on this topic, one of which was delivered twice on two consecutive days.

The focus of this one was on the use of Inman aligners and had as one of its key conclusions the statement that the process was associated with high profitability and low risk.

The other separate presentation focused on the use of Six Month Smiles and similarly intimated the same.

The first presentation suggested GDPs could charge between $£ 750-£ 1,000$ per hour for a course of treatment that would total two and a half hours, while the other recommended a total fee of between $£ 2,000-£ 3,500$ for a Six Month Smiles course of treatment.

Assuming a standard appointment time of 20 minutes for a fixed appliance adjustment every month for six months, this would total two hours of clinical time and so equate to an hourly rate of between $£ 1,000$ and $£ 1,750$ per hour.

In this regard, the veracity of the first part of the statement 'high profitability and low risk' would hold true, but at what potential cost to the profession's reputation?

Are such profit margins fair or would many regard them as excessive, if not verging on the obscene?

What about our professional obligations as defined by the General Dental Council as well? ${ }^{4}$

Could such a scale of fees lead to a challenge that a registrant may not be putting the interests of their patients ahead of their own financial gain?

As far as the second part of the statement, 'high profitability and low risk' is concerned, one need only consider the published data to learn that this is not true.

Since 2010, the number of registered complaints against GDPs using aligners has risen and at $80-90 \%$ of all aligner claims it remains significantly higher than those made against specialist orthodontists who use them. Amongst the many reasons noted for this rise, the following three are germane: inexperience and a failure to anticipate or recognise problems; failure to manage patient expectations perhaps through overselling the benefits without sufficiently stressing the risks and limitations; and overreliance on computer software treatment planning systems and remote technician aligner design and construction. ${ }^{5,6}$

So, if the statement of 'high profitability and low risk' were to be subjected to a verifiable CPD style of question to answer, the correct response would have to be that the first part of the statement is true, while the second part is false, whether or not this simply relates to the treatment process or also includes the potential for breaching regulatory professional standards.

R. A. C. Chate

The Faculty of Dental Surgery, The Royal College of Surgeons of Edinburgh

1. Maini A. Short-term cosmetic orthodontics for general dental practitioners. Br Dent J 2013; 214: 83-84

2. Chate R A C. Truth or consequences: the potential implications of short-term cosmetic orthodontics for the general dental practitioner. Br Dent J 2013; 215: 551-553.

3. Maini A, versus Chate R A C. Short-term orthodontics debate. Br Dent J 2014; 216: 386-389.

4. General Dental Council. Standards for the dental team. London: GDC, 2013.

5. Dental Protection. Brace yourself. Riskwise 2012; 42: 14.

6. Dental Protection. Capability: the dangers of short courses. Annual Review 2014: 23.

DOI: 10.1038/sj.bdj.656

\section{PHARMACOLOGY}

\section{Potency rankings}

Sir, I read with great interest the recent letter on the use of Betnovate (betamethasone valerate) cream 0.05\% in erosive oral lichen planus (OLP). ${ }^{1}$ This medication was widely used in the late 1960s until the middle 1970s, particularly in the UK, and since then has been replaced by more powerful topical corticosteroids, particularly clobetasol propionate. Unfortunately, the UK and the USA rank the potency of topical corticosteroids in different ways, which may cause some confusion. The fundamental difference is that both drug concentration and formulation are considered in the US potency ranking. As a result, Betnovate is considered potent in UK but just of medium potency in US. In practical terms, several other topical corticosteroids more powerful than betamethasone valerate (for example betamethasone dipropionate, fluocinolone, fluocinonide and fluticasone propionate) are used and published for OLP treatment. In cases recalcitrant to very potent topical corticosteroids, topical canlcineurin inhibitors such as tacrolimus, cyclosporine and pimecrolimus can be employed in different formulations. ${ }^{2-4}$

Whilst I agree with the authors that the treatment of erosive OLP can be challenging, the most outstanding advance in the last 20 years has probably been the management of the disease. The availability of topical medication such as halogenate corticosteroids and calcineruin inhibitors have permitted a huge improvement in the control of OLP lesions, even though current data are still insufficient to make strong recommendations with regard to the specific dosage, formulation, or mode of delivery or length of the therapy. However, going back 40 years does not improve the care of our OLP patients.

M. Carrozzo Newcastle-upon-Tyne

1. Kanatas A, Brotherton P. Erosive lichen planus. $\mathrm{Br}$ Dent J 2014; 216: 545 .

2. Carrozzo M, Gandolfo S. The management of oral lichen planus. Oral Dis 1999; 5: 196-205.

3. Ference J D, Last A R. Choosing topical corticosteroids. Am Fam Physician 2009; 79: 135-40.

4. Thongprasom K, Carrozzo M, Furness S, Lodi G. Interventions for treating oral lichen planus. Cochrane Database Syst Rev 2011; CD001168.

The authors A. Kanatas and P.

Brotherton respond: We read with interest the response to our letter for the use of Betnovate in the management of oral erosive lichen planus. The letter from Professor Carrozzo will be of educational value to our dental students. We agree that there are several approaches to the management of this condition and may include antimicrobials, steroids, immunomodulatory medication, topical analgesics and anti-inflammatories, barrier agents as well as laser removal. of course we would recommend the use of halogenate corticosteroids and calcineruin inhibitors in a selective group of patients. It is of interest, however, that betnovate - a medication that has been available for 40 years - is not licensed for oral mucosal membrane use. In addition there is a lack of a clinical protocol in the same way that there is a lack of an established clinical protocol in the use of calcineruin inhibitors. Moving to 'new agents' without a full understanding of the old ones is not necessarily an advancement and something to be proud of, in any specialty.

Radwan-Oczko M. Topical application of drugs used in treatment of oral lichen planus lesions. Adv Clin Exp Med 2013:22: 893-898.

DOI: $10.1038 /$ sj.bdj.2014.657 